\title{
First-Principles Investigation of Perfect and Diffuse Anti-Phase Boundaries in HCP-Based Ti-Al Alloys
}

\author{
A. van de Walle and M. Asta
}

June 7, 2006

\begin{abstract}
First-principles thermodynamic models based on the cluster expansion formalism, monte-carlo simulations and quantum-mechanical total energy calculations are employed to compute short-range-order parameters and diffuse-antiphase-boundary energies in hcp-based $\alpha$-Ti-Al alloys. Our calculations unambiguously reveal a substantial amount of SRO is present in $\alpha$-Ti- $6 \mathrm{Al}$ and that, at typical processing temperatures concentrations, the DAPB energies associated with a single dislocation slip can reach 25 $\mathrm{mJ} / \mathrm{m}^{2}$. We find very little anisotropy between the energies of DAPBs lying in the basal and prism planes. Perfect antiphase boundaries in $\mathrm{DO}_{19}$ ordered $\mathrm{Ti}_{3} \mathrm{Al}$ are also investigated and their interfacial energies, interfacial stresses and local displacements are calculated from first principles through direct supercell calculations. Our results are discussed in light of mechanical property measurements and deformation microstructure strudies in $\alpha \mathrm{Ti}-\mathrm{Al}$ alloys.
\end{abstract}

\section{Introduction}

The presence of chemical short-range order (SRO) in $\alpha$-Ti-Al was proposed over thirty years ago by Blackburn and Williams [1] to explain planar slip exhibited by alloys with concentrations greater than roughly $4 \mathrm{wt}$. $\% \mathrm{Al}(7$ at. $\% \mathrm{Al})[2,3]$. Since that time SRO has been discussed as an important factor contributing to the anomalous solid-solution strengthening of $\alpha$-Ti-Al $[1,4-6]$, as well as the low strain hardening exponents exhibited by these alloys $[3,4,7]$. Only very recently, however, has direct evidence for SRO been provided from diffuse neutron scattering measurements $[3,8]$. These measurements confirm the presence of appreciable $\mathrm{SRO}$ in Ti-6 wt. \%Al alloys, of a type consistent with the ordered $\mathrm{D}_{19}$ structure of $\alpha_{2} \mathrm{Ti}_{3} \mathrm{Al}$.

As first proposed by Fisher [9], SRO in solid solutions can provide a significant source of strengthening due to the energy cost associated with the creation of "diffuse" antiphase boundaries (DAPB) arising from shearing of the lattice by dislocation slip. The DAPB is the analogue for structures with SRO of the more familiar antiphase-boundary (APB) in a long-range-ordered crystal structure. In solid solutions with chemical SRO, the energy cost of creating a DAPB $\left(\gamma_{S R O}\right.$, defined as the excess energy per unit area of a DAPB) during slip gives rise to a friction stress on moving dislocations: $\tau_{S R O} b=\gamma_{S R O}$. A plot of the DAPB energy versus the number of shearing events, calculated from first-principles for $\alpha$-Ti-Al alloys using the approach described below, is shown in Fig. 4. This plot is qualitatively similar to theoretical results obtained for fcc-based alloy systems previously [10-13]. The energy of the DAPB after the first shear (to be referred to as $\left.\gamma_{1}\right)$ is seen to be larger than the values $\left(\gamma_{2}, \gamma_{3}\right.$, etc.) of $\gamma_{S R O}$ after multiple shearing events. As pointed out originally by Cohen and Fine [14], the second slip event in a solid-solution with SRO can partially restore the state of order, giving rise to a decrease in $\gamma_{S R O}$ (c.f. Fig. 4) and a driving force for the pairing of leading dislocations in pile-ups. Such pairing has been observed recently by transmission electronc microscopy (TEM) for dislocation pile-ups in $\alpha$-Ti-Al [15]. Fig. 4 also illustrates that after a small number of slip events the state of order is largely destroyed and subsequent dislocations experience negligible friction stress due to SRO. By this "glide-plane-softening" mechanism, SRO can promote planar slip in solid solutions, as proposed for $\alpha$-Ti-Al alloys by Blackburn and Williams [1]. 
Several TEM-based experimental studies have been undertaken to estimate the magnitude of $\tau_{S R O}$ from observed dislocation structures [3,13,16-21] in short-range-ordered alloys. To date all of this work has been devoted to fcc alloys, with the exception of the very recent experimental study by Neeraj et al. [3] for hcp-based $\alpha$-Ti-Al. In this study, magnitudes of $\gamma_{2}$ for DAPBs on prism planes were estimated from the critical stress required to form screw dislocation dipoles from elongated "hair-pin" loops, while the difference $\gamma_{1}-\gamma_{2}$ was estimated from measured spacings between paired leading edge dislocations in pile-ups. This work represents the first direct measurement of the friction stress due to SRO in $\alpha$-Ti-Al and is an important contribution to the detailed understanding of deformation properties in these alloys. The purpose of the research reported in this manuscript is to provide an independent theoretical estimate of DAPB energies in this system.

A number of theoretical studies have been undertaken to calculate the magnitude of DAPB energies for fcc and bcc alloys [10-14,22-28]. These studies have employed related methods which require (i) a description of the equilibrium state of short-range order in the alloy based upon the SRO parameters, as well as (ii) values of the interatomic interaction parameters which parametrize the energy associated with changes in atomic configurations. From the SRO parameters the number of favorable and unfavorable bonds created by a slip process can be derived and converted to an excess energy per unit area from the values of the interaction energies. To date the most comprehensive of such theoretical approaches was developed by Schwander et al. [12] who formulated general expressions for $\gamma_{S R O}$ based upon an arbitrary number of SRO parameters. For Ni-Cr alloys, Schwander et al. made use of measured SRO parameters to derive the magnitudes of pair interaction energies through the inverse-Monte-Carlo method [29]. This approach was employed more recently by Plessing et al. [13] for $\mathrm{Cu}-\mathrm{Al}$ and $\mathrm{Cu}-\mathrm{Mn}$ alloys, where good agreement was obtained between calculated values and estimates of $\gamma_{S R O}$ derived from friction stresses deduced from pile-up geometries in post-deformed samples. For $\alpha$-Ti-Al alloys, measured values of the short-range-order parameters are currently unavailable and the theoretical approach of Schwander et al. cannot be applied to the calculations DAPB energies in this system at present. An alternative approach relies upon the use of quantum-mechanical calculations to derive the values of the interatomic interaction parameters from firstprinciples [30,31]. These parameters can be employed in statistical-mechanical calculations to compute values for short-range-order parameters, allowing estimates of $\gamma_{S R O}$ to be made without the need for experimental input. Such a first-principles approach has been applied to the study of SRO hardening in $\mathrm{Cu}-\mathrm{Au}$ alloys by Mohri et al. [11]. In the present study first-principles calculations of the composition and temperature dependencies of $\gamma_{S R O}$ are undertaken for $\alpha$-Ti-Al alloys. To the best of our knowledge, this work represents the first time a calculation of DAPB energies has been undertaken for an hcp-based alloy system.

In the next section we begin by discussing first-principles results for the structure, energetics and stress of APBs in ordered $\alpha_{2} \mathrm{Ti}_{3} \mathrm{Al}$. The APB energy results serve as a basis for testing the predictive power of the calculated model interatomic interaction parameters for hcp-based $\mathrm{Ti}-\mathrm{Al}$ alloys. The next section is devoted to a detailed discussion of our study of DAPB energies. Our calculated values of $\gamma_{S R O}$ for DAPBs on prism planes are found to be consistent with the recent experimental estimates of Neeraj et al. mentioned above.

\section{Anti-Phase Boundaries}

\subsection{Methodology}

The APB energies were calculated using the local density approximation (LDA) to density functional theory (DFT), as implemented in the VASP package [32-34], which relies on ultrasoft pseudopotentials [35] and a plane wave basis. The plane wave energy cutoff was set to $270 \mathrm{eV}$ while the number of $k$-points was chosen to be at least $3500 /\left(\right.$ atom $\left.^{-1}\right)$ for all structures. ${ }^{1}$ A supercell geometry was used, where a periodic array of APBs is set up. It was verified that the APBs are sufficiently spaced from one another to avoid interactions by computing the APB energy for different supercell sizes. Separations of 5 and 16 monolayers were considered. Atomic positions were fully relaxed and the dimensions of the supercell perpendicular to

\footnotetext{
${ }^{1}$ e.g., $(3500 / n) k$-points would be selected for a structure with $n$ atom per unit cell.
} 
the plane of the APB were allowed to relax to give zero normal stress. The lattice parameters along the plane of the interface were frozen to the equilibrium lattice parameter of the $\mathrm{DO}_{19}$ structure.

The error bars we provide were derived by studying the convergence of our results with respect to plane wave energy cutoff, number of $k$-points, supercell size and the tolerance for residual forces and stresses for the numerical determination of the equilibrium geometry.

\subsection{Results and Discussion}

We consider the APB lying in the basal [0001] and prism [1100] planes, as these are the main active slip systems in Ti-Al alloys. The calculated APB energies are reported in Table 1. While there is only one type of basal APB, there are two types of prism APB, differing by the location of the APB plane, as illustrated in Fig. 1.

As shown in Table 2, the calculated APB energies are comparable to the result of earlier all-electron first-principles calculations [36], indicating that the pseudopotential approximation used provides reliable results. Our energies are systematically smaller, however, perhaps due to a more thorough treatment of the relaxations made possible by the high computational efficiency of pseudopotential-based methods.

Table 2 also shows that the APB energies calculated from first principles differ markedly from experimental estimates [37], which were obtained from measurements of the spacing between dissociated dislocations, inferring the interface energy through mechanical equilibrium considerations involving the trade-off between elastic and interfacial energy. As discussed in the next section, we estimate that the accuracy of firstprinciples calculations may be responsible for an overestimation of at most $40 \%$ of the APB energies. This error is significantly smaller that the observed disagreement with experimental estimates. The level of discrepancy between theory and experiment displayed in Table 2 has been observed for related intermetallic systems $[38,39]$. While the source of this discrepancy remains unclear, we note that the equilibrium spacings implied by our APB energies (using the same elastic constants as in [37]) are $3.0 \mathrm{~nm}, 7.0 \mathrm{~nm}$, and 1.6 $\mathrm{nm}$, respectively, for the basal, prism I, and prism II APB. These values are all very close to the minimum dislocation spacings measured by [37].

The calculated APB energies are consistent with an intuitive bond-breaking picture. The prism I, basal and prism II APB respectively create 0,1 and $2 \mathrm{Al}$-Al bonds, implying that the basal APB should have an energy than is approximately equal to the average of the prism I and prism II APB $\left(293 \mathrm{~mJ} / \mathrm{m}^{2}\right){ }^{2}$ Our calculations also provide interface stress and displacements (see Table 1). An analysis of their sign and magnitude reveals interesting features of the relaxation taking place in the vicinity of an APB in $\operatorname{Ti}_{3} \mathrm{Al}$ which may help in interpreting the stress and displacement fields that can be quantified with TEM. For the two APBs that result in a change of the nearest neighbor environment (basal and prism II), the interface contracts perpendicular to the plane of the APB $\left(d_{1}<0\right)$ and a tendency to expand along the plane of the interface $\left(\sigma_{22}>0\right.$ and $\left.\sigma_{33}>0\right)$. This behavior is reversed for the prism I APB, where no change in the nearest-neighbor environment occurs. The fact that, in all cases, the stress along the interface and the displacement perpendicular to it have opposite signs can be understood from a volume conservation argument. However, we were unable to rationalize the relative magnitudes of the stresses and displacements on the basis of intuitive atomic size considerations. This is not surprising given that such a picture is unable to describe the differences in bond length between hcp Ti and (metastable) hcp Al. According to our firstprinciples calculations, in these two pure elements, the Ti-Ti bonds are longer than $\mathrm{Al}-\mathrm{Al}$ bonds lying along the (0001) plane while the opposite is true for other nearest neighbor bonds.

\footnotetext{
${ }^{2}$ The atomic density in the plane of the APB is slightly different in basal and prism APB so that even in an ideal bond-break picture, the basal APB energy would differ slightly from the average energy of the prism APBs.
} 


\section{Diffuse Anti-Phase Boundaries}

\subsection{Methodology}

The calculation of diffuse anti-phase boundary (DAPB) energies requires the construction of a thermodynamic model of the alloy. The cluster expansion formalism $[30,31,40]$ was used to parametrize the configurational dependence of the energy of the alloy system:

$$
E(\sigma)=\sum_{i} J_{i} \sigma_{i}+\sum_{\{i, j\}} J_{i j} \sigma_{i} \sigma_{j}+\sum_{\{i, j, k\}} J_{i j k} \sigma_{i} \sigma_{j} \sigma_{k}+\ldots
$$

where $\sigma_{i}$ denotes the occupation of site $i\left(\sigma_{i}=1\right.$ for $\mathrm{Al}, \sigma_{i}=-1$ for $\mathrm{Ti}$ ) while the $J_{i \ldots}$ are effective interaction parameters to be determined from first principles and where the summations are taken over every singlet, pair, triplet,... of lattice sites. The so-called effective cluster interactions (ECI) $J_{i \ldots}$ embody all the contributions to the energetics of the alloy, including relaxations of the atoms away from their ideal lattice sites. These ECI were fitted to the energies of a set of 55 periodic structures calculated from firstprinciples using the same techniques and parameters as for the APB calculations. This procedure was carried out using the MAPS software package [41] which automates most of the tasks required to apply the cluster expansion formalism.

The ECI provide all the information we need to compute the DAPB energies. First, the ECI can be directly used to perform Monte Carlo simulations [42] that allow a first-principles computation of the phase diagram of the alloy system and degree of short-range order present in the alloy at a given temperature and composition ([43], and references therein). To this effect, we set up a Monte Carlo simulation cell consisting of a $12 \times 12 \times 6$ supercell of the hcp unit cell. The initial configuration was chosen to be random and 15000 equilibration Monte Carlo passes were performed before the short-range order was computed during an additional 2500 passes. The size of simulation cell and the number of simulation steps are such that the statistical noise in the computed short-range order parameters is orders of magnitude smaller than the error arising from the precision of the ECI themselves.

The DAPB energies $E_{D A P B}$ per unit area can then be directly expressed in terms of the ECI and the short-range order parameters obtained from the Monte Carlo simulation:

$$
E_{D A P B}=\frac{1}{A} \sum_{\{i, j\}}\left(\left\langle\sigma_{i} \sigma_{j}\right\rangle-\left\langle\sigma_{S(i)} \sigma_{S(j)}\right\rangle\right) J_{i j}+\ldots
$$

where $S$ is a function that maps a given site $i$ onto its new position, after the anti-phase boundary has been created and where $\left\langle\sigma_{i} \sigma_{j}\right\rangle$ denotes the thermodynamic and spatial average of the correlation between the occupation of sites $i$ and $j$. The sum is taken over all pairs (triplets, etc.) that are not equivalent under a translation lying in the DAPB plane which leaves the lattice invariant. $A$ is the area of a repeat unit of the plane of interest. This formulation, which is equivalent to the one introduced by Schwander [12], has the advantage that it makes the most efficient use of all the information provided by the Monte Carlo simulations. It allows us to obtain DAPB energies without having to average over various DAPB energy obtained by running a dislocation through various snapshots of the Monte Carlo cell during the simulation. This averaging is implicitly taken into account through the averaging of the correlations $\left\langle\sigma_{i} \sigma_{j}\right\rangle$ over the Monte Carlo steps and over all symmetrically equivalent clusters. As the cluster expansion does not directly provide information regarding the area $A$, we assume the disordered phase to be an hcp phase with the idea $c / a$ ratio and with an average atomic volume equal to the one of the $\mathrm{DO}_{19}$ structure. For a system with a small size mismatch ( $3 \%$, in our case), the error in $A$ introduced by this approximation will amount to at most $6 \%$.

Since we have access to the energy of a finite number of structures, we can only determine a finite number of ECI and the expansion defined by Equation (1) needs to be truncated. The choice of the optimal number of terms to keep was made through the following statistical analysis. As our goal is ultimately to predict DAPB energies, we seek to construct a cluster expansion that exhibits a good predictive power for 
alloy configurations similar to perfect APB. This exercise does not merely consists in trying many different ranges of interaction until one happens to predict APB energies that agree with the calculated ones. The agreement could be fortuitous and the predictive power of the cluster expansion for configurations that are slightly different from a perfect APB, such as a DAPB, could be poor. Instead, we calculate the statistical variance of the predicted APB energies for various choices of range of interaction, and look for the choice that minimizes that variance. The statistical variance is computed as follows. Let $J$ denote the vector of all the selected ECI and let $V$ be their estimated covariance matrix. ${ }^{3}$ The energy of a given APB, $E_{A P B}$ is related to the vector $J$ of all ECI by Equation (2), where the correlations $\left\langle\sigma_{i} \sigma_{j}\right\rangle$ are calculated for a perfect $\mathrm{DO}_{19}$ structure. This linear relationship can be written as, $E_{A P B}=C^{T} J$, where $C$ is some vector. As a result, the variance matrix of the predicted APB energy is then simply given by: $C^{T} V C$.

\subsection{Assessment of the Cluster Expansion}

The ECI of the cluster expansion that were found to minimize the sum of the statistical variances of the predicted APB energies are listed in Table 3. The ECI we calculated are very close to the ones that were determined in [44] by a fit to the experimental $\mathrm{Ti}^{-} \mathrm{Ti}_{3} \mathrm{Al}$ phase boundary. The resulting cluster expansion predicts APB energies that agree remarkably well with the calculated APB (see Table 4), given that the perfect APB energies were not used in the fit of the cluster expansion. The difference between the calculated and predicted energies is of the same order of magnitude as the error bars, confirming that our variance calculations indeed provide a very good measure of the true predictive power of the cluster expansion. A similar variance analysis will be used in the next section to estimate the errors on our calculated DAPB energies.

Although the selected range of interaction is by construction the one that will best predict the APB energies, it is still possible that longer range interactions may have an important effect on the calculated thermodynamic properties of the alloy. To investigate this concern, longer range interactions were gradually added. Adding up to 8 additional pair interactions and 6 triplet interactions changed the temperature of the $\mathrm{DO}_{19} \rightarrow$ hcp transition by less than $200 \mathrm{~K}$ and we are thus confident that our short-range cluster expansion is sufficiently accurate for our purposes.

Table 5 gives a few characteristics of our cluster expansion. The precision of the cluster expansion is quite good, as can be seen by the value of both the mean square error and the cross-validation score. ${ }^{4}$

The temperature $T_{c}$ of the $\mathrm{DO}_{19} \rightarrow$ hcp transition was calculated to be $2000 \mathrm{~K}$, which is about $40 \%$ higher than the known experimental transition temperature of $1420 \mathrm{~K}$. Such an overestimation of transition temperatures is quite common in first-principles calculations based on the local-density approximation and short-ranged cluster expansions (a list of systems where such first-principles phase diagram calculations have been undertaken can be found in [30]). To correct for this overestimation of $T_{c}$, we normalize all our temperatures by $T_{c}$. Experimental measurements should then be compared with the calculations performed at the same normalized temperature. In Fig. 2, the Ti-rich portion of the calculated phase diagram is seen to agree very well with the experimentally measured one, when both are normalized to have the same $T_{c}$.

The error in the calculated transition temperature provides us with an estimate of the error introduced by (i) the use of the Local Density Approximation in the first-principles calculations (ii) neglecting nonconfigurational sources of entropy, such as lattice vibrations and electronic entropy, and (iii) inaccuracies in the description of the configurational free energy arising from the truncation of the cluster expansion to a finite number of terms (see above). We thus expect our perfect and diffuse APB energies to be biased upward by at most $40 \%$ (this correction has neither been applied to our result nor been included in the error bars, in order to allow comparison with other first-principles results).

\footnotetext{
${ }^{3}$ Let $Y$ be an $n$-dimenstional vector and $X$ be a $n \times k$ matrix. From standard statistical analysis, the covariance matrix of a

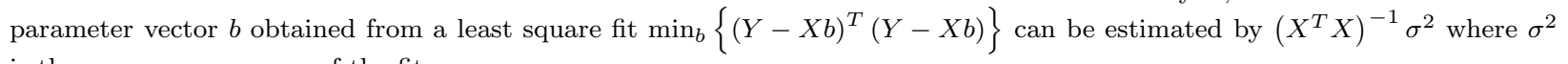
is the mean square error of the fit.

${ }^{4}$ The cross-validation score is defined to be $\left(\frac{1}{n} \sum_{s=1}^{n}\left(E_{s}-\hat{E}_{s}\right)^{2}\right)^{1 / 2}$, where $E_{s}$ is the calculated energy of structure $s$ and $\hat{E}_{s}$ is the value of the same energy predicted using the cluster expansion fitted to the remaining $n-1$ structures.
} 


\subsection{Results and Discussion}

Our first main result is that Ti-rich Ti-Al alloys do indeed exhibit a substantial amount of short-range order, as shown in Fig. 3, which plots the Warren-Cowley short-range order parameters [45] as a function of distance. This short-range order is of the $\mathrm{DO}_{19}$ type. Figures 4 and 5 show the calculated values of the DAPB energies $\gamma_{k}$ for the prism DAPB for the temperatures and concentrations marked by a cross in Fig. 2. Energies of the basal DAPB are not shown, as they are nearly identical to those for the prism DAPB, as illustrated in Fig. 6 for one specific temperature and concentration. In these plots, the abscissa $k$ represents the magnitude of the shift (in multiples of lattice vectors) between the two half crystals on each side of the DAPB, or, alternatively, the number of dislocations needed to create the DAPB. Error bars are not shown for clarity, but amount to about $15 \%$ of the calculated DAPB energies resulting from the statistical noise in the fitted ECI.

Note that the two types of prism APB can no longer be distinguished in a short-range ordered alloy because the DAPB plane cuts through various short-range ordered domains which have a random relative phase, resulting in a single average interfacial energy. The plots exhibit the characteristic oscillations that are expected from the observation that, in a perfectly ordered $\mathrm{DO}_{19}$ structure, every other dislocation would restore a perfect state of order. In a short-range ordered alloy, the ability of subsequent dislocations to restore the original state of order decreases with the number of dislocations as a result of the short-range nature of the state of order.

Our estimates of the DAPB energies are comparable to those found experimentally [3]. For $\alpha$ Ti-6 wt. $\% \mathrm{Al}$ alloys annealed at temperatures between 600 and $350^{\circ} \mathrm{C}$, experimental estimates of $\gamma_{2}$ ranged between 25 and $48 \mathrm{~mJ} / \mathrm{m}^{2}$, while a value of $6 \mathrm{~mJ} / \mathrm{m}^{2}$ was quoted for the difference $\gamma_{1}-\gamma_{2}$. Our results indicate that for an alloy of the same composition $(10$ at. $\% \mathrm{Al})$ and for the same range of relative temperature $\left(T / T_{c}\right.$ ranging from 0.45 to 0.6$), \gamma_{2}=15 \mathrm{~mJ} / \mathrm{m}^{2}$ while $\gamma_{1}-\gamma_{2}$ ranges from $7 \mathrm{~mJ} / \mathrm{m}^{2}$ to $10 \mathrm{~mJ} / \mathrm{m}^{2}$. These values of $\gamma_{2}$ are within a factor of 2 relative to experimental results, while our estimates of the difference $\gamma_{1}-\gamma_{2}$ are comparable.

An important finding is that for a substantial portion of the region of solid solubility of $\mathrm{Al}$ in $\mathrm{Ti}$, the DAPB energies $\gamma_{1}$ are larger than $2-4 \mathrm{~mJ} / \mathrm{m}^{2}$, values which have been quoted [46] as the thresholds above which transitions from homogenous to planar slip occur in fcc-based $\mathrm{Cu}-\mathrm{Zn}, \mathrm{Cu}-\mathrm{Al}$, and $\mathrm{Cu}-\mathrm{Mn}$ alloys. This result supports the proposition that the presence of SRO contributes to the predominantly planar slip in Ti-Al [1].

The fact that prism and basal DAPB have an almost identical energy is again consistent with a simple bond-breaking picture. ${ }^{5}$ Despite the small anisotropy in the average DAPB energies, the behavior of each type of DAPB under stress may differ. The local DAPB energy density may very well fluctuate as we move along the interface. For instance, a prism DAPB may contain portions that are locally analogous to a low energy prism I APB and portions that are locally similar to a high energy prism II APB, while such fluctuations should be much smaller in basal DAPB where only one type of local order exists. The average DAPB energy we compute is the quantity of interest in order to determine, for instance, the total work required to deform the material by a given amount. However, for properties such as the critical resolved shear stress (CRSS), the average may not provide enough information. A more detailed investigation of the spatial fluctuations in DAPB energy density would be needed, a topic we leave for future research.

Our calculated $\gamma_{k}$ are of the same order of magnitude as earlier theoretical studies which focused on fcc alloys [10-13]. One notable qualitative distinction is the fact that, in hcp Ti-Al alloys, the second dislocation restores the original state of short-range order to a greater extent than in these fcc alloys, as can be seen by the larger drop between $\gamma_{1}$ and $\gamma_{2}$. We attribute this difference to the fact that, in all the fcc alloys studied, the thermodynamically stable ordered phase in the vicinity of the short-range ordered region is a $\mathrm{DO}_{22}$ structure. Due to the tetragonal symmetry of this phase, different short-range ordered domains can have distinct orientations. Since the DAPB cuts through these different domains and since the $\mathrm{DO}_{22}$ has different periodicity in different directions, it is impossible for the second dislocation to restore the local

\footnotetext{
${ }^{5}$ This is not an artifact of the use of a short-range cluster expansion. The inclusion of second nearest-neighbor interactions allows the basal and prism DAPB energies to differ.
} 
order in all domains simultaneously. In contrast, in the case of Ti-Al, the hcp-based $\mathrm{DO}_{19}$ structure has the same periodicity along any [1100] direction and it follows that the second dislocation can partially restore the original local order in all domains simultaneously.

\section{Conclusion}

Our first main result is the confirmation, on the basis of first-principles calculations, that $\mathrm{DO}_{19}$-type shortrange is present in the hcp $\alpha$-Ti phase of the Ti-Al alloy. We have also calculated the energy of diffuse antiphase boundaries (DAPB). We find that for an alloy at a composition of 10 at. $\%$ of $\mathrm{Al}$ and a temperature between 600 and $300^{\circ} \mathrm{C}$, a single dislocation creates a DAPB with an interfacial energy ranging from 22 to $25 \mathrm{~mJ} / \mathrm{m}^{2}$ while a second dislocation creates a DAPB having an interfacial energy of $15 \mathrm{~mJ} / \mathrm{m}^{2}$. These values are significantly higher than $4 \mathrm{~mJ} / \mathrm{m}^{2}$, a value estimated to be sufficient to induce a transition from homogeneous to planar slip in fcc-based alloys [46].

The computer programs used to complete this work can be obtained by contacting the authors. These include an easy-to-use Monte Carlo code that provides, among other quantities, short-range order parameters and automatic phase boundary plotting capabilities. A companion code implements the Schwander et al. [12] formalism to its full generality, allowing the calculation of DAPB of any orientation, for any lattice type, and for any number of short-range order parameter.

\section{Acknowledgements}

We are grateful for numerous helpful discussions with Dr. T. Neeraj and Prof. M. Mills from The Ohio State University who suggested this problem, and who provided us with a copy of the dissertation cited in Ref. [3]. This research was supported by the National Science Foundation under program DMR-0080766. 


\section{References}

[1] M. J. Blackburn and J. C. Williams, Trans. ASM 62, pp. 398-399 (1969).

[2] J. Y. Lim, J. McMahon, D. P. Pope, and J. C. Williams, Metall. Trans. 7A, pp. 139-144 (1976).

[3] T. Neeraj, Ph.D. thesis, The Ohio State University, 2000.

[4] H. R. Ogden, D. J. Maykuth, W. L. Finley, and R. I. Jaffee, Trans. AIME 197, pp. 267-272 (1953).

[5] H. W. Rosenberg and W. D. Nix, Metall. Trans. 4, pp. 1333-1342 (1972).

[6] H. Conrad, Scripta Metall. 7, pp. 509-512 (1973).

[7] T. Sakai and M. E. Fine, Scripta Metall. 8, pp. 541-544 (1974).

[8] T. Neeraj, J. L. Robertson, and M. J. Mills (unpublished).

[9] J. C. Fisher, Acta Metall. 2, pp. 9-10 (1954).

[10] T. Mohri, D. de Fontaine, and J. M. Sanchez, Metall. Trans. 17A, pp. 189-194 (1986).

[11] T. Mohri, T. Tsutsumi, O. Sasaki, and K. Watanabe, Metall. Trans. 21A, pp. 3165-3169 (1990).

[12] P. Schwander, B. Schönfeld, and G. Kostorz, Phys. Stat. Sol. (b) 172, pp. 73-85 (1992).

[13] J. Plessing, C. Achmus, H. Neuhauser, B. Schönfeld, and G. Kostorz, Z. Metallkd. 88, pp. 630-635 (1997).

[14] J. B. Cohen and M. E. Fine, Acta Metall. 11, pp. 1106-1115 (1963).

[15] T. Neeraj, D.-H. Hou, G. S. Daehn, and M. J. Mills, Acta Mater. 48, pp. 1225-1238 (2000).

[16] F. Prinz, H. P. Karnthaler, and H. O. K. Kirchner, Acta Metall. 29, pp. 1029-1036 (1981).

[17] H. Neuhaüser, O. B. Arkan, and H. H. Potthoff, Mater. Sci. Eng. 81, pp. 201-209 (1986).

[18] N. Clement, D. Caillard, and J. L. Martin, Acta. Metall. 32, pp. 961-975 (1984).

[19] J. Olfe and H. Neuhäuser, phys. stat. sol. (a) 109, pp. 149-160 (1988).

[20] M. Jouiad, N. Clement, and A. Coujou, Phil. Mag. A 77, pp. 689-699 (1998).

[21] M. Jouiad, F. Pettinari, N. Clement, and A. Coujou, Phil. Mag. A 79, pp. 2591-2602 (1999).

[22] P. A. Flinn, Acta Metall. 6, pp. 631-635 (1958).

[23] J. B. Cohen and M. E. Fine, J. Phys. et Radium 23, pp. 749-762 (1962).

[24] R. O. Williams, Acta Metall. 18, pp. 457-466 (1970).

[25] A. R. Büchner and W. Pitsch, Z. Metallkd. 76, pp. 651-656 (1985).

[26] G. Inden, S. Bruns, and H. Ackermann, Phil. Mag. A 53, pp. 87-100 (1986).

[27] W. Pitsch, Scripta Metall. 8, pp. 813-819 (1974).

[28] W. Pitsch, Scripta Metall. 9, pp. 1059-1062 (1975).

[29] V. Gerold and J. Kern, Acta Metall. 35, pp. 393-399 (1987).

[30] D. de Fontaine, Solid State Phys. 47, pp. 33-176 (1994). 
[31] A. Zunger, in NATO ASI on Statics and Dynamics of Alloy Phase Transformation, edited by P. E. Turchi and A. Gonis (Plenum Press, New York, 1994), Vol. 319, pp. 361-405.

[32] G. Kresse and J. Furthmüller, Vienna Ab-initio Simulation Package (VASP).

[33] G. Kresse and J. Furthmüller, Phys. Rev. B 54, pp. 11169-11186 (1996).

[34] G. Kresse and J. Furthmüller, Comp. Mat. Sci. 6, pp. 15-50 (1996).

[35] D. Vanderbilt, Phys. Rev. B 41, pp. 7892-7895 (1990).

[36] C. L. Fu, J. Zou, and M. H. Yoo, Scripta Metall. Mater. 33, pp. 885-891 (1995).

[37] M. Legros, A. Couret, and D. Caillard, Phil. Mag. A 73, pp. 61-80 (1996).

[38] M. Asta and A. A. Quong, Phil. Mag. Lett. 76, pp. 331-339 (1997).

[39] M. H. F. Sluiter, Y. Hashi, and Y. Kawazoe, Comput. Mater. Sci. 14, pp. 283-290 (1999).

[40] J. M. Sanchez, F. Ducastelle, and D. Gratias, Physica 128A, pp. 334-350 (1984).

[41] A. van de Walle and G. Ceder, MIT Ab-initio Phase Stability (MAPS) code, available by contacting avdw@alum.mit.edu.

[42] K. Binder and D. W. Heermann, Monte Carlo Simulation in Statistical Physics (Springer-Verlag, New York, 1988).

[43] C. Wolverton, V. Ozoliņš, and A. Zunger, J. Phys.: Condens. Matter 12, pp. 2749-2768 (2000).

[44] M. Shimono and H. Onodera, Phys. Rev. B 61, pp. 14271-14274 (2000).

[45] B. E. Warren, X-Ray Diffraction (Addison-Wesley, Reading, MA, 1969).

[46] H. Roelofs, B. Schönfeld, G. Kostorz, and W. Buhrer, phys. stat. sol. (b) 187, pp. 31-42 (1995).

[47] U. R. Kattner, J.-C. Lin, and Y. A. Chang, Metall. Trans. A 23A, pp. 2081-2090 (1992). 


\section{Tables}

\begin{tabular}{|r|r|r|r|}
\hline & \multicolumn{1}{|c|}{ basal } & \multicolumn{1}{c|}{ prism I } & \multicolumn{1}{c|}{ prism II } \\
\hline$\gamma\left(\mathrm{mJ} / \mathrm{m}^{2}\right)$ & $249 \pm 5$ & $108 \pm 2$ & $477 \pm 5$ \\
\hline$\sigma_{22}\left(\mathrm{~J} / \mathrm{m}^{2}\right)$ & $1.4 \pm 0.2$ & $-1.5 \pm 0.2$ & $3.8 \pm 0.2$ \\
\hline$\sigma_{33}\left(\mathrm{~J} / \mathrm{m}^{2}\right)$ & $0.8 \pm 0.2$ & $-0.1 \pm 0.2$ & $0.3 \pm 0.2$ \\
\hline$d_{1}(\AA)$ & $-0.02 \pm 0.01$ & $0.16 \pm 0.01$ & $-0.10 \pm 0.01$ \\
\hline$d_{3}(\AA)$ & $-0.15 \pm 0.03$ & 0 & 0 \\
\hline
\end{tabular}

Table 1: Calculated Interface Energy $\gamma$, Interface Stress $\sigma$ and Displacement $d$ of the Basal and Prism APB. Stresses and displacements are reported in cartesian coordinate system where the two first basis vectors are respectively defined by the unit normal and the Burgers vector of the APB. Compressive stresses are positive. The negative sign of $d_{3}$ indicates that $\mathrm{Al}-\mathrm{Al}$ bonds at the interface are shortened by the displacements. All unreported stesses and displacements are zero by symmetry.

\begin{tabular}{|r|r|r|r|}
\hline & \multicolumn{3}{|c|}{$\gamma\left(\mathrm{mJ} / \mathrm{m}^{2}\right)$} \\
\hline & basal & prism I & prism II \\
\hline present & 249 & 108 & 477 \\
\hline first-principles [36] & 300 & 133 & 506 \\
\hline experiment [37] & 63 & 42 & 84 \\
\hline
\end{tabular}

Table 2: Comparison between calculated and Measured Interface Energy $\gamma$ of the Basal and Prism APB.

\begin{tabular}{|c|r|r|}
\hline Pair & Interaction $(\mathrm{eV})$ & Ref [44] \\
\hline$(0,0,0)-\left(\frac{2}{3}, \frac{1}{3}, \frac{1}{2}\right)$ & $0.057 \pm 0.005$ & 0.0588 \\
\hline$(0,0,0)-(1,0,0)$ & $0.047 \pm 0.003$ & 0.0588 \\
\hline$(0,0,0)-\left(\frac{2}{3},-\frac{2}{3}, \frac{1}{2}\right)$ & $-0.014 \pm 0.004$ & -0.0235 \\
\hline
\end{tabular}

Table 3: ECI of the Cluster Expansion. Coordinates are expressed in the conventional hexagonal coordinate system. Error bars are one standard deviation wide. 


\begin{tabular}{|l|l|l|l|}
\hline$\gamma\left(\mathrm{mJ} / \mathrm{m}^{2}\right)$ & basal & prism I & prism II \\
\hline supercell & $249 \pm 5$ & $108 \pm 2$ & $477 \pm 5$ \\
\hline clus. exp. & $234 \pm 34$ & $71 \pm 20$ & $427 \pm 55$ \\
\hline
\end{tabular}

Table 4: APB Energies Obtained from Supercell Calculations and Predicted from a Cluster Expansion. Error bars on the predicted energies are one standard deviation wide.

\begin{tabular}{|l|l|}
\hline Number of structures: & 55 \\
\hline Atom/unit cell: & $2-12$ \\
\hline Mean square error: & $0.031 \mathrm{eV} /$ atom \\
\hline Cross-validation score: & $0.034 \mathrm{eV} /$ atom \\
\hline Predicted $T_{c}$ of $\mathrm{DO}_{19} \rightarrow \mathrm{hcp}:$ & $2000 \mathrm{~K} \pm 25 \mathrm{~K}$ \\
\hline Experimental $T_{c}$ of $\mathrm{DO}_{19} \rightarrow \mathrm{hcp}:$ & $1420 \mathrm{~K}$ \\
\hline
\end{tabular}

Table 5: Characteristics of the Cluster Expansion. 


\section{Figures}

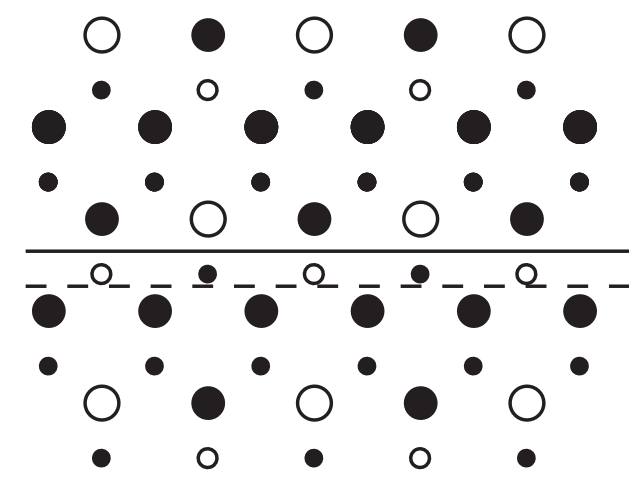

Figure 1: $\mathrm{DO}_{19}$ structure and the location of prism I (dashed line) and prism II (solid line) planes. The size of the circles distinguishes distinct (0001) planes of atom. Al atoms are shown in white, Ti atoms in black.

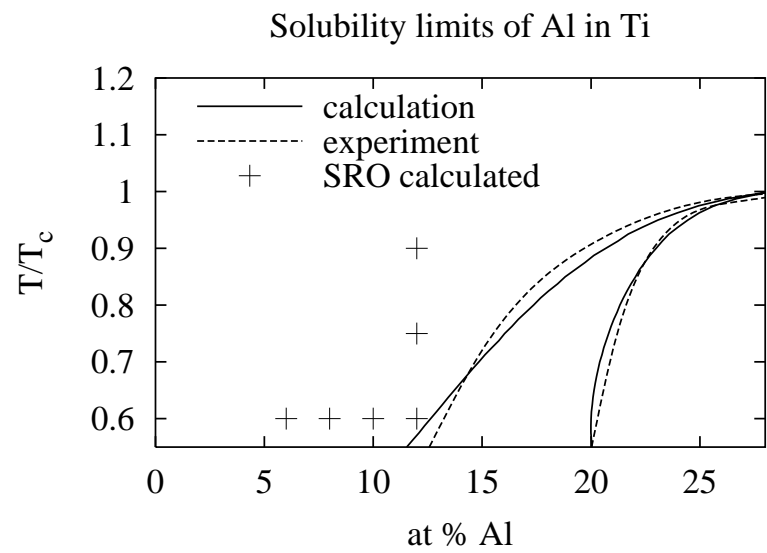

Figure 2: Calculated and Experimental Phase Boundaries (from [47]). Also shown are the points where the DAPB energies were calculated. 


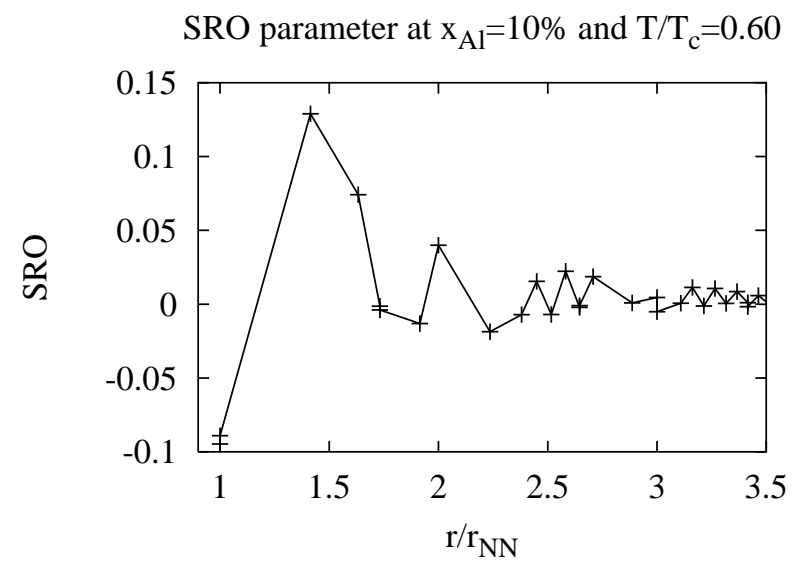

Figure 3: Calculated Warren-Cowley short-range order parameter as a function of distance (normalized by nearest neighbor distance).

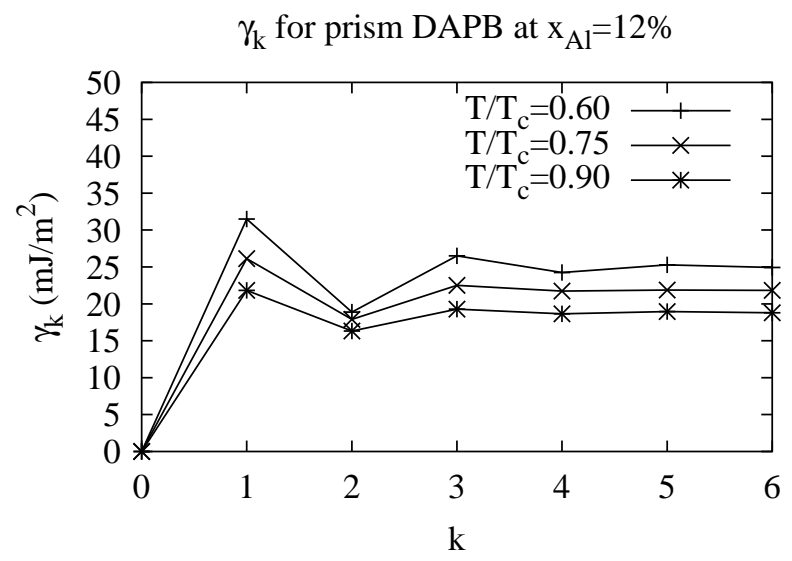

Figure 4: Calculated Prism DAPB Energies as a Function of Temperature.

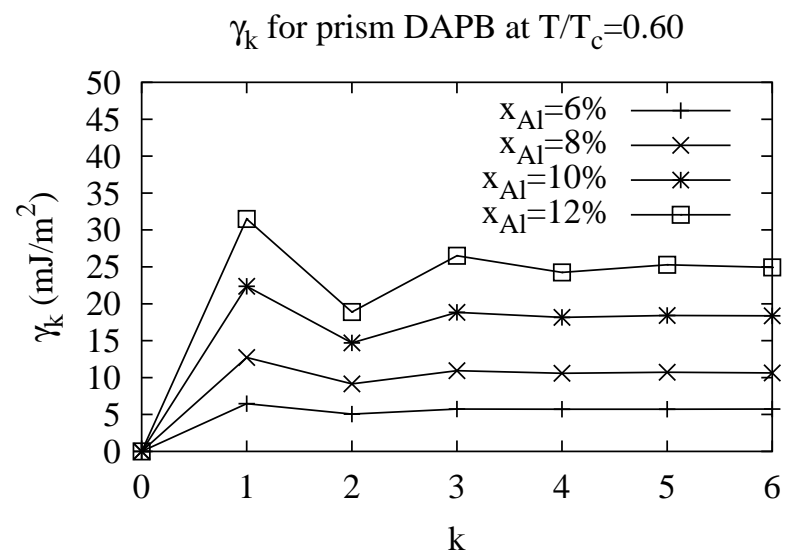

Figure 5: Calculated Prism DAPB Energies as a Function of Concentration. 


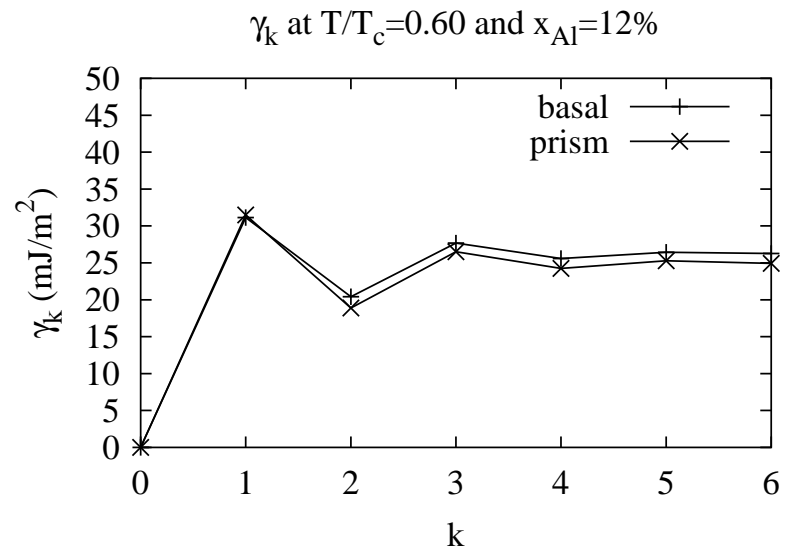

Figure 6: Calculated Prism and basal DAPB Energies. 


\section{Figure Captions}

Figure 1. $\mathrm{DO}_{19}$ structure and the location of prism I (dashed line) and prism II (solid line) planes. The size of the circles distinguishes distinct (0001) planes of atom. Al atoms are shown in white, Ti atoms in black.

Figure 2. Calculated and Experimental Phase Boundaries (from [47]). Also shown are the points where the DAPB energies were calculated.

Figure 3. Calculated Warren-Cowley short-range order parameter as a function of distance (normalized by nearest neighbor distance).

Figure 4. Calculated Prism DAPB Energies as a Function of Temperature.

Figure 5. Calculated Prism DAPB Energies as a Function of Concentration.

Figure 6. Calculated Prism and basal DAPB Energies. 